\title{
Aplicación de somatotropina recombinante porcina en lechones durante la primera semana de vida: efecto sobre variables metabólicas y somatométricas
}

\author{
Porcine recombinant somatotropin administered to piglets during the first week \\ of life: effects on metabolic and somatometric variables
A Olmos-Hernández ${ }^{\mathrm{a}}$, ME Trujillo-Ortega ${ }^{\mathrm{b}}, \mathrm{M}^{\mathrm{A}}$ Alonso-Spilsbury ${ }^{\mathrm{c}}$, M Becerril-Herrera ${ }^{\mathrm{d}}$, R Hernández-González ${ }^{\mathrm{e}}$, D Mota-Rojas ${ }^{\mathrm{e}^{*}}$

\author{
aPrograma de Doctorado en Ciencias Biológicas, Universidad Autónoma Metropolitana-Xochimilco, México DF, México. \\ bProducción Animal: Cerdos, Universidad Nacional Autónoma de México, Ciudad Universitaria, México DF, México. \\ 'Universidad Autónoma Metropolitana-Xochimilco, Departamento de Producción Agrícola y Animal, Área de Investigación: \\ Ecodesarrollo de la Producción Animal, México DF, México. \\ dEIAH-Benemérita Universidad Autónoma de Puebla, Domicilio conocido, San Juan Acateno, Municipio Teziutlán, Puebla, México. \\ eDepartamento de Investigación Experimental y Bioterio, Instituto Nacional de Ciencias Médicas y \\ Nutrición Salvador Zubirán, México DF, México.
}

\section{SUMMARY}

\begin{abstract}
The aim of this study was to evaluate the effect of porcine recombinant somatotropin (rpST) administered to piglets during their first week of life, on metabolic and somatometric variables. A total of 220 piglets born from 40 hybrid Landrace x Large White sows were used. Piglets were randomly assigned to either Group $1\left(\mathrm{G}_{1}\right.$, Control, $\left.\mathrm{n}=106\right)$ or Group $2\left(\mathrm{G}_{2}\right.$, rpST treatment, $\left.\mathrm{n}=114\right)$. The rpST dose was $1 \mathrm{mg} / \mathrm{kg}$ every $24 \mathrm{~h}$ during 7 consecutive days. Serum $\mathrm{pH}$ at birth showed significant differences between groups $(7.42 \pm 0.47$ and $7.31 \pm 0.59)$, although this difference dissapeared on day 8 . There was a significant increase $(\mathrm{P}<0,001)$ in plasma glucose levels for the treated group on day $8(68.78 \pm 10.44$ and $103.71 \pm 18.44)$. Average daily weight gain was also higher for the somatotropin treated group compared to the control (137.25 \pm 31.31 and $209.21 \pm 45.97)$. Comparisons for interaction between treatment and sex showed dramatic differences $(P<0,001)$ for glucose, lactate and weight gain for the males from $\mathrm{G}_{2}$ when compared to the females from the same experimental group on day 7 post-treatment. Piglets treated with rpST showed significant differences in abdominal perimeter ( $28.67 \pm 4.41$ and $31.79 \pm 3.74)$, dorsal length $(28.42 \pm 2.66$ and $30.21 \pm 4.70)$, and ham roundness (18.79 \pm 2.62 and $22.38 \pm 2.98)$. There is an ongoing research that will allow to determine the composition of body mass (bone or muscle) in the treated piglets.
\end{abstract}

Palabras clave: somatotropina recombinante porcina, lechón, vitalidad neonatal, peso.

Key words: porcine recombinant somatotropin, piglet, neonatal vitality, weight.

\section{INTRODUCCIÓN}

Actualmente se busca uniformar el peso de los lechones al nacimiento o durante la lactancia para reducir la mortalidad en esta etapa y aumentar el peso del lechón destetado. El aumento de peso al destete contribuye a disminuir el retraso del crecimiento, la mortalidad postdestete y, en última instancia, disminuye la edad a peso de mercado (Harrell y col 1997, Kim y col 2001). En la producción de carne existe una amplia gama de estrategias de la biotecnología disponible para modificar el balance entre la carne magra y el tejido adiposo en el crecimiento y la deposición (Villanueva-García y col 2006). Intentos recientes para mejorar la eficiencia de la producción y el crecimiento en animales han llevado al uso de citrato del

Aceptado: 18.11.2009.

* Universidad Autónoma Metropolitana Calzada del Hueso 1100, Col, Villaquietud, México, D.F.; dmota100@yahoo.com.mx sildenafil (Sánchez-Aparicio y col 2008) y somatotropina exógena (Spicer y col 1992, Braña y col 2001, Trujillo-Ortega y col 2006). Asimismo, existe una base de datos cada vez mayor que apoya el uso de somatotropina recombinante (rpST) o derivado pituitario como un agente que mejora la eficiencia del crecimiento y la composición de la canal en los cerdos (Solomon y col 1997, Braña y col 2001). Varios estudios han demostrado que la administración de la pST exógena a los cerdos mejora significativamente la tasa de crecimiento, la eficiencia de la utilización de nutrientes y el rendimiento en canal (Cromwell y col 1992, Solomon y col 1997), al disminuir la acumulación del tejido adiposo y al aumentar los depósitos de proteína (Louveau y Ethertont 1992, Braña y col 2001). Esto sugiere que la pST pueda tener efectos directos en las fibras musculares o, alternativamente, que la ST regule la síntesis de IGF-I en el musculoesquelético de cerdos en crecimiento (Louveau y Ethertont 1992). Sin embargo, son escasos los estudios que se han realizado sobre la aplicación de rpST en neonatos porcinos, con la intención de mejorar los indicadores de 
morbilidad y mortalidad en la etapa predestete. El objetivo del presente estudio fue evaluar el efecto de la aplicación de rpST sobre variables metabólicas y somatométricas durante la primera semana de vida en lechones.

\section{MATERIAL Y MÉTODOS}

El experimento fue desarrollado en una granja porcina, con un sistema de producción intensiva ubicada en el Estado de Hidalgo, México, de acuerdo con los lineamientos de ética del uso de animales (Sherwin y col 2003). El estudio fue aprobado por la Comisión del Doctorado en Ciencias Biológicas de la Universidad Autónoma Metropolitana, Campus Xochimilco, México DF, México, así como por el Comité de Investigación en Animales (CINVA) del Instituto Nacional de Ciencias Médicas y Nutrición Salvador Zubirán México DF, México (Registro CINVA: 219).

\section{ANIMALES}

Los animales recibieron atención veterinaria a lo largo del estudio y los procedimientos de uso y cuidado de los animales se condujeron de acuerdo a la NOM-062-ZOO(Diario Oficial de la Federación 1999). Un total de 221 lechones nacidos de 40 cerdas híbridas Landrace x Large White se incluyeron en el estudio. Las cerdas fueron monitoreadas 48 horas previas a la fecha probable de parto y fueron sincronizadas con $10 \mathrm{mg}$ de prostaglandinas $\left(\mathrm{PGF}_{2} \alpha\right)$ vía intramuscular $24 \mathrm{~h}$ previas a su fecha probable de parto. Durante el parto las cerdas y los lechones fueron atendidos por dos de los investigadores.

La distribución de los lechones en dos grupos se realizó por medio de un muestreo aleatorio simple, el cual consistió en utilizar una tabla de números aleatorios para identificar los individuos que se incluyeron en cada grupo y reducir las posibilidades de un sesgo. El Grupo 1 estuvo conformado por 106 lechones $\left(G_{1}\right.$, Testigo) de los cuales 62 eran hembras y 44 machos enteros, el Grupo 2 lo conformaron un total de 114 lechones $\left(\mathrm{G}_{2}\right.$, tratado con pST), 55 hembras y 59 machos enteros. Los machos se mantuvieron enteros a lo largo del estudio. Las variables propuestas para el estudio incluyeron peso corporal $(\mathrm{kg})$, calificación de la vitalidad al nacimiento, sexo, glucosa $(\mathrm{mg} / \mathrm{dL})$, lactato $(\mathrm{mg} / \mathrm{dL}), \mathrm{pH}$, ganancia diaria de peso $(\mathrm{g}) \mathrm{y}$ mediciones somatométricas $(\mathrm{cm})$.

\section{COLECCIÓN DE LAS MUESTRAS SANGUÍNEAS}

Las muestras de sangre de los lechones fueron colectadas del seno venoso retroorbital, inmediatamente después del nacimiento, procedimiento aprobado por la regulación mexicana NOM-062-ZOO-1999.

Los niveles sanguíneos de glucosa $(\mathrm{mg} / \mathrm{dL})$, lactato $(\mathrm{mg} / \mathrm{dL})$ y $\mathrm{pH}$ fueron medidos por medio de un analizador automático (GEM Premier 3000, Instrumentation Laboratory Diagnostics, Italia), procedimiento validado en estudios previos con lechones recién nacidos (Trujillo-Ortega y col 2007, Orozco-Gregorio y col 2008). Inmediatamente después de la obtención de la muestra de sangre, los lechones fueron pesados en una báscula digital (Salter Weight-Tronix Ltd., West Bromwich, United Kingdom).

\section{VITALIDAD AL NACIMIENTO}

Al nacimiento, la vitalidad de los lechones se evaluó de acuerdo a la escala descrita por Zaleski y Hacker (1993) y modificada por Mota-Rojas y col (2005).

\section{PARÁMETROS SOMATOMÉTRICOS}

Para determinar el ritmo de crecimiento en los lechones se realizaron varias mediciones morfométricas por medio de un vernier y cinta métrica. Éstas se realizaron al nacimiento y al término del octavo día. Las mediciones corporales fueron: ancho de la cabeza $(\mathrm{cm})$, ancho de la grupa $(\mathrm{cm})$, perímetro torácico $(\mathrm{cm})$, perímetro abdominal $(\mathrm{cm})$, largo dorsal $(\mathrm{cm})$ y redondez del jamón $(\mathrm{cm})$.

\section{APLICACIÓN DE RPST}

El grupo de lechones tratado con $1 \mathrm{mg} / \mathrm{kg}$ de $\mathrm{rpST}$ obtuvieron la primera dosis (previamente pesados) 24 horas después de nacidos y así consecutivamente cada veinticuatro horas durante siete días que duró el experimento. El grupo testigo recibió solución salina como placebo; la vía de administración fue intramuscular en la tabla del cuello (músculo braquicefálico).

\section{ANÁLISIS ESTADÍSTICO}

Los datos son presentados como medias y desviación estándar. Los resultados obtenidos fueron analizados mediante un diseño completamente al azar con covariable cuyo modelo fue el siguiente:

$$
\begin{aligned}
& Y_{i j k}=\mu+t_{i}+\beta\left(X_{i}-\bar{X}\right)+\xi_{i j k} \\
& i=1,2 \\
& j=1,2
\end{aligned}
$$

Donde:

$\mathrm{Y}_{\mathrm{ijk}}=$ Variable respuesta

$\mu=$ Media general

$\mathrm{u}_{\mathrm{i}}=$ Efecto del tratamiento

$\beta=$ Coeficiente de regresión

$\mathrm{X}_{\mathrm{i}}=$ Covariable

$\overline{\mathrm{X}}=$ Media general de la covariable

$\xi_{\mathrm{ijk}}=$ Error aleatorio en la repetición $\mathrm{k}$, nivel j de B y nivel i de A. 
Para determinar la existencia de diferencias significativas entre tratamientos de las medias ajustadas, se utilizó la prueba ajustada de Tuckey-Cramer.

Las variables respuesta se analizaron estadísticamente usando los siguientes procedimientos:

Comparación de tratamientos mediante prueba de t para muestras independientes. El criterio de prueba fue una $\mathrm{t}$ de Student.

$t=\frac{\left(\overline{X_{1}}-\overline{X_{2}}\right)-\left(\mu_{1}-\mu_{2}\right)}{S_{\overline{X_{1}}-\overline{X_{2}}}} \approx t-$ Student

Donde:

$\overline{X_{1}}-\overline{X_{2}}=$ Diferencia de medias muestrales.

$\mu_{1}-\mu_{2}=0$, bajo Ho.

$S^{2} \overline{X_{1}}-\overline{X_{2}}=S_{P}^{2}(1 / n 1+1 / n 2)$

$S_{p}^{2}=\frac{\left(n_{1}-1\right) S_{1}^{2}+\left(n_{2}-1\right) S_{2}^{2}}{\left(n_{1}-1\right)+\left(n_{2}-1\right)}$

$n_{1} \mathrm{y} n_{2}=$ Tamaño de las muestras.

$S_{2}^{1}, S_{2}^{2}=$ Varianza de las observaciones en cada muestra.

Evaluación del efecto del sexo mediante el modelo propuesto. Para determinar la existencia de diferencias significativas entre tratamientos se utilizó la prueba de Tuckey $(\mathrm{P}<0,05)$.
Análisis de variables $p H$ y vitalidad mediante estadística no paramétrica. Se utilizaron las pruebas de U de Mann Whitney y Kruskal Wallis, respectivamente.

En la elaboración de los análisis estadísticos se utilizó el programa SAS ver 9.0 para Windows (2004).

\section{RESULTADOS Y DISCUSIÓN}

En el cuadro 1 se presentan los resultados de los diferentes indicadores evaluados, tanto al nacimiento como a la semana de vida. Con respecto a la variable peso al nacimiento, hubo una diferencia significativa $(\mathrm{P}<0,05)$ entre grupos; sin embargo, al incluir el peso como covariable se trató de eliminar la diferencia existente en el peso de los lechones al nacimiento, tratando de igualarlos para poder observar el verdadero efecto de la somatotropina. A la semana de tratamiento el fenómeno se invirtió, observándose significativamente $(\mathrm{P}<0,001)$ un mayor peso en los lechones del $\mathrm{G}_{2}$. Otra respuesta de gran importancia es la concentración de glucosa en sangre, no se apreciaron diferencias significativas en ambos grupos al nacimiento; sin embargo, a la semana de vida se distingue un incremento significativo $(\mathrm{P}<0,001)$ del $50 \%$ en la concentración de los niveles de glucosa plasmática $(\mathrm{mg} / \mathrm{dL})$ en los lechones del grupo tratado con $\operatorname{rpST}\left(\mathrm{G}_{1}: 68,78 \pm 10,44 ; \mathrm{G}_{2}: 103,71 \pm 18,44\right)$. Dunshea $y$ col (1992) reportan que el tratamiento con pST aumenta los niveles circulantes de glucosa, efecto que se observa en los cerdos que fueron tratados con la hormona en nuestro estudio, en donde a la semana de tratamiento se observó

Cuadro 1. Comparación de las variables peso, vitalidad, glucosa, lactato y pH observados en lechones del grupo testigo y tratados con rpST al nacimiento y a los 8 días de edad (media \pm DE).

Weight, vitality, glucose, lactate and $\mathrm{pH}$ observed in the control and rpST treated piglets at birth and on day 8 (mean \pm standard deviation).

\begin{tabular}{|c|c|c|c|c|c|c|}
\hline & \multicolumn{3}{|c|}{ Nacimiento } & \multicolumn{3}{|c|}{ Día 8} \\
\hline & $\begin{array}{c}\mathrm{G}_{1} \\
\text { Grupo Testigo } \\
\text { Placebo } \\
\mathrm{n}=106\end{array}$ & $\begin{array}{c}\mathrm{G}_{2} \\
\text { Grupo } \\
\text { Somatotropina } \\
\mathrm{n}=114\end{array}$ & & $\begin{array}{c}\mathrm{G}_{1} \\
\text { Grupo Testigo } \\
\text { Placebo } \\
\mathrm{n}=106\end{array}$ & $\begin{array}{c}\mathrm{G}_{2} \\
\text { Grupo } \\
\text { Somatotropina } \\
\mathrm{n}=114\end{array}$ & \\
\hline Variables & \multicolumn{2}{|c|}{ Media \pm DE } & $\mathrm{P}$ & \multicolumn{2}{|c|}{ Media $\pm \mathrm{DEr}$} & $\mathrm{P}$ \\
\hline Peso (g) & $1.459,1 \pm 128,93$ & $1.393,8 \pm 148,37$ & 0,0006 & $2.551,1 \pm 276,61$ & $3.067,5 \pm 414,92$ & $<0,0001$ \\
\hline Vitalidad* & $8,0 \pm 3,0$ & $8,0 \pm 4,0$ & 0,0574 & - & - & - \\
\hline Glucosa (mg/dL) & $68,73 \pm 12,53$ & $69,52 \pm 11,27$ & 0,6229 & $68,78 \pm 10,44$ & $103,71 \pm 18,44$ & $<0,0001$ \\
\hline Lactato (mg/dL) & $37,89 \pm 6,83$ & $38,57 \pm 7,71$ & 0,4893 & $29,43 \pm 6,80$ & $31,57 \pm 7,34$ & 0,0265 \\
\hline $\mathrm{pH}^{*}$ & $7,42 \pm 0,47$ & $7,31 \pm 0,59$ & 0,0025 & $7,46 \pm 0,26$ & $7,45 \pm 0,30$ & 0,4418 \\
\hline Ganancia de peso (g) & - & - & - & $1.098 \pm 250,55$ & $1.673,7 \pm 367,81$ & $<0,0001$ \\
\hline Ganancia diaria de peso $(\mathrm{g})$ & - & - & - & $137,25 \pm 31,31$ & $209,21 \pm 45,97$ & $<0,0001$ \\
\hline
\end{tabular}

Los datos son presentados en medias y desviación estándar. Las variables se compararon entre los grupos por una prueba de t-Student Kruskal-Wallis $(\mathrm{P}<0,001)$.

*Expresados como media \pm rango y analizados con la prueba U de Mann Whitney. 
una elevación de los niveles sanguíneos de glucosa de 1,5 veces más en los lechones del grupo tratado con la hormona. Sin embargo, Wilson y col (2008) concluyen que los cerdos tratados con pST no mostraron una reducción significativa de glucosa en comparación con los animales control. Respecto del pH sanguíneo se aprecian diferencias significativas $(\mathrm{P}<0,05)$ entre grupos al nacimiento $(7,42 \pm 0,47$ y 7,31 $\pm 0,59)$, proceso que no sucede a la semana de tratamiento. Con relación a los indicadores ganancia diaria de peso (GDP) y la ganancia de peso total (GP) a la semana de aplicación diaria de la rpST, se observaron diferencias significativas $(\mathrm{P}<0,001)$ entre grupos, distinguiendo una marcada ganancia de peso diario, mayor para el grupo tratado $(209,21 \mathrm{~g} \pm 45,97)$ respecto al grupo testigo $(137,25 \mathrm{~g} \pm 31,31)$, por consiguiente la ganancia de peso total fue mayor para este mismo grupo. La administración de rpST durante la primera semana de vida a dosis de $1 \mathrm{mg} / \mathrm{kg}$ en lechones causó un incremento del $52 \%$ en la ganancia diaria de peso, y por consiguiente una mayor ganancia de peso total, demostrando que los lechones del grupo tratado fueron $20 \%$ más pesados que los lechones del grupo testigo. Se desconoce si dicho peso se debe a un aumento en la masa ósea o muscular o grasa. Es probable que los lechones a los que se les aplicó rpST ganaron más peso debido a que la $\mathrm{GH}$ promueve la síntesis de proteínas, a través del efecto indirecto mediado por el IGF-I (el principal regulador del crecimiento somático), que es sintetizado por el hígado como efecto de la GH (Hardy
1994). Los lechones tratados con rpST fueron $6 \%$ más largos que los del grupo testigo. Juul y col (1995) reportan que la administración de GH conlleva a la elevación de IGF-I y proteína fijadora de IGF (IGFBP-3), y dentro de la gran variedad de actividades metabólicas en células y tejidos de la IGF-I, participa en la regulación del desarrollo perinatal (Nonoshita y col 1994, Leal-Guadarrama y col 2002).

En el cuadro 2 destacan las diferencias de peso entre machos enteros y hembras del grupo testigo, lo mismo sucedió con el grupo tratado. No obstante no hubo diferencias significativas en las hembras del grupo testigo comparadas con los machos enteros del grupo experimental. Conviene señalar que en esta variable los machos del $G_{1}$ fueron significativamente $(1.508,86 \pm 132,01)$ más pesados que las hembras de su mismo grupo $(1.423,70 \pm 115,17)$, y que los machos enteros y hembras $(1.431,69 \pm 173,80$, $1.353,09 \pm 101,81$ respectivamente) del $\mathrm{G}_{2}$.

Los valores de glucosa y lactato fueron significativamente superiores para los machos de ambos grupos respecto de las hembras; sin embargo, al comparar los valores de lactato entre animales de diferente sexo del mismo grupo, sí se aprecian diferencias significativas $(\mathrm{P}<0,001)$.

$\mathrm{Al}$ analizar los resultados de la calificación de vitalidad que presentaron los lechones al nacimiento se puede apreciar que las hembras de ambos grupos presentaron una calificación significativamente mayor $(\mathrm{P}<0,001)$ en comparación con los machos de sus respectivos grupos. No obstante, al comparar los animales del mismo sexo

Cuadro 2. Efecto del sexo en las variables evaluadas en lechones al nacimiento del grupo testigo y tratados con rpST (media \pm DE). Effects of gender on evaluated variables from control and treated newborn piglets with rpST (mean $\pm \mathrm{DE})$.

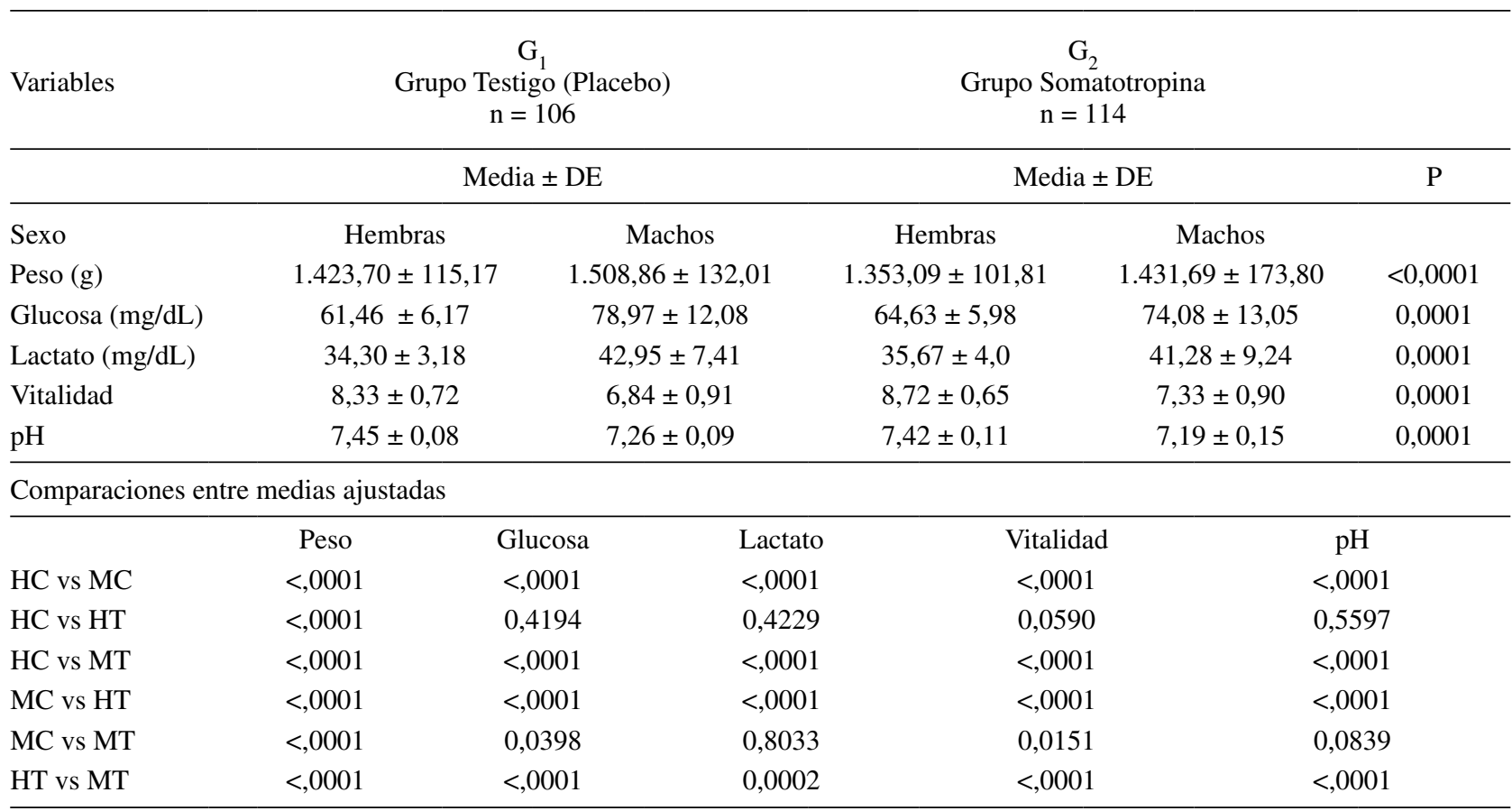

$\mathrm{HC}=$ Hembras Control, $\mathrm{MC}=$ Machos Control, $\mathrm{HT}=$ Hembras Tratadas, $\mathrm{MT}=$ Machos Tratados. 
entre grupos no se observan diferencias significativas. Llama la atención que los machos enteros del grupo testigo obtuvieron una calificación significativamente $(\mathrm{P}<0,001)$ más baja $(6,84 \pm 0,91)$ respecto a las hembras $(8,33 \pm 0,72)$ de su mismo grupo, y a los machos enteros y hembras $(7,33 \pm 0,90: 8,72 \pm 0,65$, respectivamente $)$ del grupo rpST. Con relación a la variable $\mathrm{pH}$ hubo diferencias significativas entre grupos.

Al analizar los resultados de las diferentes variables evaluadas una semana después del nacimiento y posterior a la aplicación de la rpST durante este periodo (cuadro 3), se puede apreciar que los machos enteros del $\mathrm{G}_{2}$ fueron significativamente $(\mathrm{P}<0,001)$ más pesados $(3.330,50 \pm 392,60)$ respecto a las hembras de su mismo grupo $(2.785,27 \pm 194,94)$, así como a los machos enteros y hembras del $\mathrm{G}_{1}(2.668,18 \pm 266,74 ; 2.478,22 \pm 257,53)$. Con relación a las diferencias significativas en pesos por efecto de la interacción tratamiento y sexo, cabe destacar que las hembras tratadas fueron $12 \%$ más pesadas que las hembras del grupo testigo, en tanto que los machos enteros del grupo tratado pesaron $24 \%$ más que los machos enteros del grupo testigo. Los machos tratados con la $\operatorname{rpST}\left(\mathrm{G}_{2}\right)$ fueron significativamente más pesados que las hembras tratadas. Los resultados pueden explicarse debido a que el crecimiento, densidad e integridad de los huesos dependen de una adecuada función de un grupo de hormonas, dentro de las cuales se encuentran los esteroides sexuales (estrógenos, progesterona, testosterona), siendo bien sabido que las hormonas sexuales regulan el metabolismo óseo (Sánchez y col 2006). La testosterona estimula la proliferación de las células del hueso y la diferenciación y, por tanto, tiene un efecto positivo sobre el metabolismo óseo (Kasperk y col 1997). En contraste, los estrógenos atenúan la acción de la $\mathrm{GH}$, inhibiendo la función endocrina a través del hígado (Meinhardt y Ho 2006). Lo anterior puede explicar las diferencias observadas entre sexos de los lechones tratados con rpST.

Por otro lado, los niveles de glucosa fueron significativamente diferentes $(\mathrm{P}<0,001)$ entre animales del mismo grupo y entre ambos grupos. Los machos tratados con rpST presentaron niveles de glucemia más elevada $(112,20 \pm 20,46)$ con respecto a los no tratados. Los niveles de lactato plasmático fueron significativamente $(\mathrm{P}<0,001)$ mayores en los machos tratados $(33,25 \pm 8,41)$ respecto a las hembras tratadas y a las del grupo no tratadas. No se distinguen diferencias significativas con los machos $G_{1}$. De igual forma estas diferencias $(\mathrm{P}<0,001)$ fueron también observadas entre machos y hembras del grupo no tratado.

Cuadro 3. Efecto del sexo sobre las variables observadas en lechones del grupo control y tratados con rpST a los 8 días de edad (media $\pm \mathrm{DE})$.

Effects of gender on observed variables from control and treated piglets with rpST on day 8 (mean $\pm \mathrm{DE})$.

\begin{tabular}{|c|c|c|c|c|c|}
\hline \multirow[t]{2}{*}{ Variables } & \multicolumn{2}{|c|}{$\begin{array}{c}\mathrm{G}_{1} \\
\text { Grupo Testigo (Placebo) } \\
\mathrm{n}=106\end{array}$} & \multicolumn{2}{|c|}{$\underset{\mathrm{n}=114}{\mathrm{G}_{2}}$} & \\
\hline & \multicolumn{2}{|c|}{ Media \pm DE } & \multicolumn{2}{|c|}{ Media \pm DE } & $\mathrm{P}$ \\
\hline Sexo & Hembras & Machos & Hembras & Machos & \\
\hline Peso (g) & $2.478,22 \pm 257,53$ & $2.668,18 \pm 266,18$ & $2.785,27 \pm 194,94$ & $3.330,50 \pm 392,60$ & $<0,0001$ \\
\hline Glucosa (mg/dL) & $65,64 \pm 6,88$ & $73,20 \pm 12,84$ & $94,60 \pm 9,94$ & $112,20 \pm 20,46$ & $<0,0001$ \\
\hline Lactato (mg/dL) & $27,54 \pm 4,61$ & $32,09 \pm 8,39$ & $29,78 \pm 5,52$ & $33,23 \pm 8,41$ & 0,0001 \\
\hline $\mathrm{pH}$ & $7,45 \pm 0,06$ & $7,46 \pm 0,06$ & $7,44 \pm 0,06$ & $7,45 \pm 0,07$ & 0,9477 \\
\hline Ganancia de peso $(\mathrm{g})$ & $1.054,51 \pm 257,88$ & $1.159,31 \pm 228,84$ & $1.432,18 \pm 209,05$ & $1.898,81 \pm 340,60$ & $<0,0001$ \\
\hline Ganancia diaria de peso (g/día) & $131,81 \pm 32,23$ & $144,91 \pm 28,60$ & $179,02 \pm 26,13$ & $237,35 \pm 42,57$ & $<0,0001$ \\
\hline
\end{tabular}

Comparaciones entre medias ajustadas

\begin{tabular}{lcccccc}
\hline & Peso & Glucosa & Lactato & pH & GP & GDP \\
\hline HC vs MC & 0,1306 & 0,0307 & 0,0061 & 0,9998 & 0,1306 & 0,1306 \\
HC vs HT & $<, 0001$ & $<, 0001$ & 0,3184 & 0,9928 & $<, 0001$ & $<, 0001$ \\
HC vs MT & $<, 0001$ & $<, 0001$ & $<, 0001$ & 0,9409 & $<, 0001$ & $<, 0001$ \\
MC vs HT & 0,0002 & $<, 0001$ & 0,3929 & 0,9986 & 0,0002 & 0,0002 \\
MC vs MT & $<, 0001$ & $<, 0001$ & 0,8493 & 0,9740 & $<, 0001$ & $<, 0001$ \\
HT vs MT & $<, 0001$ & $<, 0001$ & 0,0428 & 0,9930 & $<, 0001$ & $<, 0001$ \\
\hline
\end{tabular}

$\mathrm{HC}=$ Hembras Control, $\mathrm{MC}=$ Machos Control, HT $=$ Hembras Tratadas, $\mathrm{MT}=$ Machos Tratados 
Durante el transcurso del experimento los machos del $\mathrm{G}_{2}$ obtuvieron una mayor GDP $(237,35 \pm 42,57 \mathrm{~g} / \mathrm{d})$; este resultado fue significativamente mayor $(\mathrm{P}<0,001)$ comparado con las hembras $\mathrm{G}_{2}(179,02 \pm 26,13 \mathrm{~g} / \mathrm{d})$, así como con las hembras y machos enteros del grupo testigo $(131,81 \pm 32,23 ; 144,91 \pm 30,13, \mathrm{~g} / \mathrm{d}$ respectivamente $)$. El crecimiento de los cerdos se puede observar en el cuadro 4 .

En las variables somatométricas evaluadas en los lechones al nacimiento no se observaron diferencias significativas entre los $\mathrm{G}_{1}$ y $\mathrm{G}_{2}$; sin embargo, a la semana de edad, una vez que se administró rpST diariamente, sí hubo diferencias significativas entre grupos en las variables: perímetro abdominal $\left(\mathrm{G}_{1}: 28,67 \pm 4,41 \mathrm{~cm}\right.$ Vs. $\left.\mathrm{G}_{2}: 31,79 \pm 3,74 \mathrm{~cm}\right)$, perímetro torácico $\left(\mathrm{G}_{1}\right.$ : $32,65 \pm 4,46 \mathrm{~cm} \mathrm{Vs.} \mathrm{G}_{2}: 35,70 \pm 4,32 \mathrm{~cm}$ ) largo dorsal $\left(\mathrm{G}_{1}: 28,42 \pm 2,66 \mathrm{~cm} \mathrm{Vs} . \mathrm{G}_{2}: 30,21 \pm 4,70 \mathrm{~cm}\right)$, y redondez del jamón $\left(\mathrm{G}_{1}: 18,79 \pm 2,62 \mathrm{~cm} \mathrm{Vs.} \mathrm{G}_{1}: 22,38 \pm 2,98 \mathrm{~cm}\right)$. Estudios realizados por Braña y col (2001) señalan que la ST aplicada a cerdos de engorda está directamente relacionada con el mayor consumo de alimento en climas templados. Rehfeldt y col (2001) indican que el tratamiento de cerdas con ST durante la gestación temprana tuvo un efecto significativo en el incremento de peso de órganos abdominales y vísceras en los fetos; por ejemplo, el tracto intestinal y estómago se incrementaron en un 26 y $15 \%$, respectivamente, en tanto que los órganos torácicos, los pulmones y corazón incrementaron su peso en un 14 y 7\%, respectivamente. Asimismo, Braña y col (2001) encontraron que el peso del corazón e hígado en cerdos finalizados tratados con ST se incrementaron significativamente en un 12 y $25 \%$, respectivamente. Lo anterior probablemente explica el porqué el perímetro abdominal y el torácico se incrementaron en el grupo tratado. Respecto al incremento del diámetro del jamón en un 19\%, que es la tendencia que evidenció el grupo tratado con rpST, está relacionada directamente con la mayor deposición de proteína muscular.

\section{CONCLUSIONES}

La administración de rpST durante la primera semana de vida a dosis de $1 \mathrm{mg} / \mathrm{kg}$ en lechones recién nacidos que permanecen con su madre produjo un incremento del $52 \%$ en la ganancia diaria de peso, y por consiguiente una mayor ganancia de peso total, demostrando que los lechones son $20 \%$ más pesados. Asimismo, este grupo de recién nacidos presentó hiperglucemia y un mejor desarrollo corporal. En futuros estudios será importante demostrar si los lechones más pesados son más vigorosos y modifican su comportamiento durante la lactancia mejorando la conversión alimenticia en esta etapa, y si el incremento de las variables morfométricas está relacionado con el incremento de peso de los huesos y órganos en las cavidades torácica y abdominal. Asimismo, será interesante demostrar cómo cambia la relación músculo-grasa-hueso por efecto de la aplicación de ST con respecto del peso corporal.

\section{RESUMEN}

Con el fin de evaluar el efecto de la administración de la somatotropina recombinante porcina (rpST) en lechones, sobre las variables metabólicas y somatométricas durante la primera semana de vida, se evaluaron un total de 220 lechones al nacimiento de 40 cerdas híbridas Landrace $\mathrm{x}$ Large White. Los lechones fueron clasificados aleatoriamente en dos grupos. El Grupo 1 conformado por 106 lechones (Grupo testigo) y el grupo 2 por un total de 114 lechones (Grupo tratado con pST). La

Cuadro 4. Comparación entre los parámetros somatométricos observados en lechones al nacimiento y tratados con rpST durante la primera semana de vida (media $\pm \mathrm{DE}$ ).

Somatometric parameters observed in newborn and 8 days old piglets treated with rpST during the first week of life (mean \pm standard deviation).

\begin{tabular}{|c|c|c|c|c|c|c|}
\hline \multirow{3}{*}{ Variables } & \multicolumn{2}{|c|}{ Nacimiento } & & \multicolumn{2}{|c|}{ Día 8} & \\
\hline & $\begin{array}{c}\mathrm{G}_{1} \\
\text { Grupo } \\
\text { Testigo }\end{array}$ & $\begin{array}{c}\mathrm{G}_{2} \\
\text { Grupo } \\
\text { Somatotropina }\end{array}$ & & $\begin{array}{c}\mathrm{G}_{1} \\
\text { Grupo } \\
\text { Testigo }\end{array}$ & $\begin{array}{c}\mathrm{G}_{2} \\
\text { Grupo } \\
\text { Somatotropina }\end{array}$ & \\
\hline & Media \pm DE & Media $\pm \mathrm{DE}$ & $\mathrm{P}$ & Media $\pm \mathrm{DE}$ & Media $\pm \mathrm{DE}$ & $\mathrm{P}$ \\
\hline Ancho de la cabeza $(\mathrm{cm})$ & $5,78 \pm 0,37$ & $5,82 \pm 0,41$ & 0,5857 & $6,65 \pm 0,43$ & $6,69 \pm 0,53$ & 0,6286 \\
\hline Ancho de la grupa (cm) & $6,40 \pm 0,60$ & $6,32 \pm 0,61$ & 0,4972 & $8,10 \pm 0,91$ & $8,91 \pm 1,06$ & 0,7158 \\
\hline Perímetro torácico $(\mathrm{cm})$ & $26,70 \pm 3,26$ & $26,47 \pm 3,41$ & 0,7157 & $32,65 \pm 4,46$ & $35,70 \pm 4,32$ & 0,0004 \\
\hline Perímetro abdominal $(\mathrm{cm})$ & $22,09 \pm 1,92$ & $22,43 \pm 2,14$ & 0,3661 & $28,67 \pm 4,41$ & $31,79 \pm 3,74$ & 0,0001 \\
\hline Largo dorsal (cm) & $22,68 \pm 2,28$ & $22,55 \pm 1,72$ & 0,7383 & $28,42 \pm 2,66$ & $30,21 \pm 4,70$ & 0,0131 \\
\hline Redondez del jamón (cm) & $13,55 \pm 1,19$ & $13,36 \pm 1,20$ & 0,4191 & $18,79 \pm 2,62$ & $22,38 \pm 2,98$ & $<0,0001$ \\
\hline
\end{tabular}

La comparación entre el Grupo Testigo y el Grupo Tratado se realizó mediante la prueba de t-Student. 
administración de la rpST se realizó a una dosis de $1 \mathrm{mg} / \mathrm{kg}$ cada 24 h durante siete días. En el $\mathrm{pH}$ al nacimiento se apreciaron diferencias significativas $(\mathrm{P}<0,05)$ en ambos grupos $(7,42 \pm 0,47$ y 7,31 $\pm 0,59)$, fenómeno que desaparece a la semana de vida. A la semana se observó un aumento significativo $(\mathrm{P}<0,001)$ en los niveles de glucosa en los lechones del $G_{2}$ vs. $G_{1}(103,71 \pm 18,4468,78 \pm 10,44)$. Al final del experimento la ganancia diaria de peso fue mayor para el grupo tratado con rpST $(209,21 \pm 45,97)$ respecto al grupo testigo $(137,25 \pm 31,31)$. $\mathrm{Al}$ comparar el efecto del tratamiento por sexo se observaron marcadas diferencias significativas $(\mathrm{P}<0,001)$ en el $\mathrm{pH}$, glucosa, lactato y peso ganado en los machos del $\mathrm{G}_{2}$ con respecto a las hembras del mismo grupo a los siete días postratamiento. Los lechones tratados con rpST presentaron diferencias significativas en las variables: perímetro abdominal ( $28,67 \pm 4,41$ y $31,79 \pm 3,74$ respectivamente), largo dorsal $(28,42 \pm 2,66$ y $30,21 \pm 4,70$ respectivamente) y redondez del jamón $(18,79 \pm 2,62$ y $22,38 \pm 2,98$ respectivamente). Investigación en proceso permitirá distinguir la masa corporal ganada por los lechones, en ósea o muscular.

\section{AGRADECIMIENTOS}

El presente estudio es parte de los avances de la Tesis Doctoral de la primera autora, en el Programa de Doctorado en Ciencias Biológicas en la Universidad Autónoma Metropolitana Iztapalapa-Xochimilco. Se agradece la beca de Doctorado del CONACYT que recibe Adriana Olmos-Hernández.

\section{REFERENCIAS}

Braña D, L Ángeles, R Loeza, A Ángeles, J Cuarón. 2001. Somatotropina recombinante en la finalización de cerdos en dos condiciones climáticas. Tec Pec Mex 39, 215-228.

Cromwell GL, TS Stahly, LA Edgerton, HJ Monegue, TW Burnell, BC Schenck, BR Schricker. 1992. Recombinant porcine somatotropin for sows during late gestation and throughout lactation. J Anim Sci 70, 1404-1416.

Diario Oficial de la Federación. 1999. Especificaciones técnicas para la producción, cuidado y uso de los animales de laboratorio. NOM062-ZOO. Norma Oficial Mexicana, México.

Dunshea FR, DE Bauman, RD Boyd, AW Bell. 1992. Temporal response of circulating metabolites and hormones during somatotropin treatment of growing pigs. J Anim Sci 70, 123-131.

Hardy RN. 1994. Fisiología del sistema endocrino. Editorial El Manual Moderno, México.

Harrell RJ, MJ Thomas, RD Boyd, SM Czerwinski, NC Steele, DE Bauman. 1997. Effect of porcine somatotropin administration in young pigs during the growth phase from 10 to 25 kilograms. J Anim Sci 73, 3152-3160.

Juul A, P Dalgaard, WG Blum, P Bang, K Hall, KF Michaelsen, J Muller, NE Skakkebaek. 1995. Serum levels of insulin-like growth factor (IGF)-binding proteins (IGFBP-3) in healthy infants, children, and adolescents: the relation to IGF-1, IGF-2, IGFBP-1, IGFBP-2, age, sex, body mass index, and pubertal maturation. $J$ Clin Endocrinol Metab 80, 2534-2542.

Kasperk CH, G Wakley, T Hierl, R Ziegler. 1997. Gonadal and adrenal androgens are potent regulators of human bone cell metabolism in vitro. J Bone Miner Res 12, 464-471.

Kim SW, RA Easter, WL Hurley. 2001. The regression of unsuckled mammary glands during lactation in sows: the influence of lactation stage, dietary nutrients, and litter size. J Anim Sci 79, 2659-2668.

Leal-Guadarrama LI, M Ochoa-Rojas, JD Méndez. 2002. Importancia clínica de los factores de crecimiento parecidos a la insulina. Gac Méd Méx 139, 589-599.

Louveau I, TD Ethertont, 1992. Characterization of somatotropin binding sites in pig skeletal muscle. J Anim Sci 70, 1801-1805.

Martínez JA, PJ Buttery, JT Pearson. 1984. The mode of action of anabolic agents, the effects of testosterone on growth rates and muscle protein metabolism in female rats. Br J Nut, 54, 515-521.
Meinhardt, UJ, KKY Ho. 2006. Modulation of growth hormone action by sex steroids. Clin Endocrinol 65, 413-422.

Mota-Rojas D, J Martínez-Burnes, ME Trujillo-Ortega, A López, A Rosales, R Ramírez, H Orozco, A Merino, ML Alonso-Spilsbury. 2005. Uterine and fetal asphyxia monitoring in parturient sows treated with oxytocin. Anim Reprod Sci 86, 131-141.

Nonoshita LD, NC Wathen, BA Dsupin, T Chard, LC Giudice. 1994. Insulin-like growth factors (IGFs), IGF-binding proteins (IGFBPs), and proteolyzed IGFBP-3 in embryonic cavities in early human pregnancy: their potential relevance to maternal-embryonic and fetal interactions. J Clin Endocrinol Metab 79, 1249-1255.

Orozco-Gregorio H, D Mota-Rojas, M Alonso-Spilsbury, A OlmosHernández, R Ramírez-Necoechea, EY Velázquez-Armenta, AA Nava-Ocampo, R González-Hernández, ME Trujillo-Ortega, D Villanueva-García. 2008. Short-term neurophysiologic consequences of intrapartum asphyxia in piglets born by spontaneous parturition. Int J Neurosci 118, 1299-1315.

Rehfeldt C, G Kuhn, G Nürnberg, E Kanitz, F Schneider, M Beyer, K Nürnberg, K Ender. 2001. Effects of exogenous somatotropin during early gestation on maternal performance, fetal growth and compositional traits in pigs. J Anim Sci 79, 1789-1799.

Sánchez JC, DA Navarro, OA Hernández. 2006. Acción fisiopatológica integrada de las hormonas sobre el tejido óseo. Rev Cubana Endocrinol 17, 1-9.

Sánchez-Aparicio P, D Mota-Rojas, AA Nava-Ocampo, ME TrujilloOrtega, A Alfaro-Rodríguez, E Arch, M Alonso-Spilsbury. 2008. Effects of sildenafil on the fetal growth of guinea pigs and their ability to survive induced intrapartum asphyxia. Am J Obstet Gynecol 198, 127-133.

SAS Institute Inc. 2004. Version 9.0. Cary, NC, USA: SAS Institute, Inc.

Sherwin CM, SB Christiansen, IJ Duncan, HW Erhard, DC Lay, JA Mench, CE O'Connor, JC Petherick. 2003 Guidelines for the ethical use of animals in applied ethology studies. Appl Anim Behav Sci 81 291-305.

Solomon MB, VG Pursel, RG Campbelld, NC Steele. 1997. Biotechnology for porcine products and its effect on meat products. Food Chem 59, 499-504.

Spicer LJ, J Klindtt, FC Buonomo, RM Maurert, JT Yen, SE Echternkamp. 1992. Effect of porcine somatotropin on number of granulosa cell luteinizing hormone/hurnan chorionic gonadotropin receptors, oocyte viability and concentrations of steroids and insulin-like growth factors I and II in follicular fluid of lean and obese gilts. J Anim Sci 70, 3149-3157.

Trujillo-Ortega ME, D Mota-Rojas, R Hernández-González, Y Velázquez-Armenta, AA Nava-Ocampo, R Ramírez-Necoechea, M Becerril-Herrera, M Alonso-Spilsbury. 2006. Obstetric and neonatal outcomes to recombinant porcine somatotropin administered in the last third of pregnancy to primiparous sows. J Endcrinol 189, 575-582.

Trujillo-Ortega ME, D Mota-Rojas, A Olmos-Hernández, M Alonso-Spilsbury, M González, H Orozco, R Ramírez-Necoechea, AA Nava-Ocampo. 2007. A study of piglets born by spontaneous parturition under uncontrolled conditions: could this be a naturalistic model for the study of intrapartum asphyxia? Acta Biomed 78, 29-35.

Villanueva-García D, A Olmos-Hernández, D Mota-Rojas, M GonzálezLozano ME Trujillo-Ortega, B Acosta, DL Reyes, R Ramírez, M Alonso-Spilsbury. 2006. Effects of recombinant porcine somatotropin on pig fetal growth and metabolism: a review. Am J Biochem Biotech 2, 129-137.

Wilson FA, RA Orellana, A Suryawan, HV Nguyen, AS Jeyapalan, J Frank TA Davis. 2008. Stimulation of Muscle Protein Synthesis by Somatotropin in Pigs Is 2 Independent of the SomatotropinInduced Increase in Circulating Insulin. Am J Physiol Endocrinol Metab 295, 876-883.

Zaleski HM, RR Hacker. 1993. Variables related to the progress of parturition and probability of stillbirth in swine. Can Vet J 34, 109-113. 